\title{
ON THE SUBMETACOMPACTNESS OF PRODUCTS
}

\author{
YUKINOBU YAJIMA \\ (Communicated by Dennis K. Burke) \\ Dedicated to Professor Yukihiro Kodama on his 60th birthday
}

\begin{abstract}
In this paper, we raise the question of whether the product $X \times Y$ of a regular space $X$ with a $\sigma$-closure-preserving cover by compact sets and a submetacompact space $Y$ is submetacompact, and we show that the product $X \times Y$ is weakly submetacompact.
\end{abstract}

\section{QUESTION AND ITS BACKGROUND}

All spaces dealt with here are topological spaces. Regular spaces are assumed to be $T_{1}$. No separation axioms are assumed, unless stated otherwise.

The question raised here is the following:

Question 1.1. Let $X$ be a regular space with a $\sigma$-closure-preserving cover by compact sets. Is $X \times Y$ submetacompact for every submetacompact space $Y$ ?

In this paper, we will give a partial answer to this question.

Let $\mathscr{V}$ be a collection of subsets of a space $X$ and $x$ a point of $X$. We denote by $\operatorname{ord}(x, \mathscr{V})$ the number of members of $\mathscr{V}$ which contain $x$. For a cover $\mathscr{U}$ of $X, \mathscr{V}$ is a refinement of $\mathscr{U}$ if $\mathscr{V}$ is a cover of $X$ and each member of $\mathscr{V}$ is contained in some member of $\mathscr{U}$.

Definition 1.2. A space $X$ is said to be submetacompact (= $\theta$-refinable) [WW] if for every open cover $\mathscr{U}$ of $X$ there is a sequence $\left\{\mathscr{V}_{n}\right\}$ of open refinements of $\mathscr{U}$ such that for each $x \in X$ one can choose some $n<\omega$ with $\operatorname{ord}\left(x, \mathscr{V}_{n}\right)<\omega$.

The concept of submetacompactness is well known and has been studied in many papers. However, the submetacompactness of products has been hardly discussed. One of the reasons for this seems to be the following example of Przymusiński [P].

Example 1.3. There are a separable metric space $X$ and a first countable, separable, regular Lindelöf space $Y$ such that $X \times Y$ is not submetacompact.

Received by the editors November 24, 1987 and, in revised form, May 20, 1988.

1980 Mathematics Subject Classification (1985 Revision). Primary 54B10, 54D18.

Key words and phrases. Submetacompact, weakly submetacompact, $\sigma$-closure-preserving cover, topological game, winning strategy. 
Recently, Burke [B, §6] dealt with the submetacompactness of products, and stated the following result.

Theorem 1.4. Let $X$ be a regular $\Sigma$-space and $Y$ a P-space. If $X$ and $Y$ are paracompact Hausdorff (regular Lindelöf, subparacompact, metacompact, submetacompact), then $X \times Y$ is paracompact (Lindelöf, subparacompact, metacompact, submetacompact).

The paracompact case and the Lindelöf case were proved in [N], and the subparacompact case in [L]. The metacompact case was stated in the below of [B, Corollary 6.26] and in Remark to [ $Y_{1}$, Proposition 2.2]. The submetacompact case was proved by Burke himself there.

However, in Theorem 1.4, the space $Y$ is a $P$-space. Of course, it is desirable that no conditions on $Y$ are assumed except the covering property. The following is easily obtained (for example, see [B, Theorem 6.1].

Proposition 1.5. Let $X$ be a regular $\sigma$-compact space. If $Y$ is a paracompact Hausdorff (regular Lindelöf, subparacompact, metacompact, submetacompact) space, then $X \times Y$ is paracompact (Lindelöf, subparacompact, metacompact, submetacompact).

Let $\mathscr{C}$ be a collection of closed sets in a space $X$. Recall that $\mathscr{C}$ is closurepreserving if $\cup \mathscr{C}^{\prime}$ is closed in $X$ for each $\mathscr{C}^{\prime} \subset \mathscr{C}$, and that $\mathscr{C}$ is $\sigma$-closurepreserving if $\mathscr{C}=\bigcup_{n<\omega} \mathscr{C}_{n}$ with each $\mathscr{C}_{n}$ closure-preserving.

Proposition 1.5, except the submetacompact case, has been extended as follows.

Theorem 1.6. Let $X$ be a regular space with a $\sigma$-closure-preserving cover by compact sets. If $X$ and $Y$ are paracompact Hausdorff (regular Lindelöf, subparacompact, metacompact) spaces, then $X \times Y$ is paracompact (Lindelöf, subparacompact, metacompact).

The paracompact case and the Lindelöf case were proved in [T], and the subparacompact case and the metacompact case in $\left[\mathrm{Y}_{2}\right]$.

Note that a space $X$ with a $(\sigma)$-closure-preserving cover by compact sets is metacompact (submetacompact) (cf. [K, PJ]). It follows easily from the metacompact case of Theorem 1.6 that if $X$ is the space described in Theorem 1.6, then $X \times Y$ is submetacompact for every metacompact space $Y$. Moreover, for the submetacompact case, the following was proved in $\left[Y_{2}\right]$.

Theorem 1.7. If $X$ is a regular space with a $\sigma$-closure-preserving cover by compact sets, then $X \times Y$ is submetacompact for every subparacompact space $Y$.

In view of Theorem 1.6, Question 1.1 seems to be a natural question.

Remark 1.8. In Question 1.1, we may assume that the space $X$ has a closurepreserving cover by compact sets, since the countable union of submetacompact closed subspaces is also submetacompact. 


\section{A PARTIAL ANSWER TO THE QUESTION}

The following concept, which is weaker than that of submetacompactness, is also well known.

Definition 2.1. A space $X$ is said to be weakly submetacompact (= weakly $\theta$ refinable) [BL] if every open cover $\mathscr{U}$ of $X$ has an open refinement $\bigcup_{n<\omega} \mathscr{V}_{n}$ such that for each $x \in X$ one can choose some $n<\omega$ with $0<\operatorname{ord}\left(x, \mathscr{V}_{n}\right)<$ $\omega$.

Let $D C$ be the class of regular spaces which have a discrete cover by compact sets. The topological game $G(D C, X)$ is in the sense of Telgársky [T].

Definition 2.2. Let $X$ be a space. We say that Player I has a winning strategy in the game $G(D C, X)$ (cf. [T, GT]) if there is a function $s$ from $2^{X}$ into $2^{X} \cap D C$, where $2^{X}$ is the family of all closed sets in $X$, satisfying

(i) $s(F) \subset F$ for each $F \in 2^{X}$,

(ii) if $\left\{F_{n}\right\}$ is a decreasing sequence of closed sets in $X$ such that $s\left(F_{n}\right) \cap$ $F_{n+1}=\varnothing$ for each $n<\omega$, then $\bigcap_{n<\omega} F_{n}=\varnothing$.

The following is our main result, which is proved in the next section.

Theorem 2.3. Let $X$ be a regular submetacompact space. If Player I has a winning strategy in the game $G(D C, X)$, then $X \times Y$ is weakly submetacompact for every weakly submetacompact space $Y$.

By [T, Corollary 10.2] and Theorem 2.3, we obtain the following partial answer to Question 1.1.

Corollary 2.4. If a regular space $X$ has a $\sigma$-closure-preserving cover by compact sets, then $X \times Y$ is weakly submetacompact for every (weakly) submetacompact space $Y$.

Moreover, by [T, Theorem 9.7] and Theorem 2.3, we have

Corollary 2.5. If a regular space $X$ is subparacompact and $C$-scattered, then $X \times Y$ is weakly submetacompact for every (weakly) submetacompact space $Y$.

\section{PROOF OF THEOREM}

Let $X \times Y$ be a product. A subset of the form $U \times V$ in $X \times Y$ is called a rectangle. For a rectangle $R$ in $X \times Y, R^{\prime}$ and $R^{\prime \prime}$ denote the projections of $R$ into $X$ and $Y$, respectively, that is, $R=R^{\prime} \times R^{\prime \prime}$. We say that a rectangle $R$ is open (closed $\times$ open) if $R^{\prime}$ is open (closed) in $X$ and $R^{\prime \prime}$ is open in $Y$ (cf. $\left.\left[\mathrm{Y}_{2}\right]\right)$.

For a finite sequence $a=\left(\alpha_{1}, \ldots, \alpha_{n}\right)$, let $a \oplus \alpha=\left(\alpha_{1}, \ldots, \alpha_{n}, \alpha\right)$ for $n \geq 1$, and $a_{-}=\left(\alpha_{1}, \ldots, \alpha_{n-1}\right)$ for $n \geq 2$. In particular, $(\alpha)_{-}=\varnothing$.

We denote by $\omega^{<\omega}$ the set of all finite sequences consisting of natural numbers. Moreover, $\left(\omega^{<\omega}\right)^{<\omega}$ denotes the set of all finite sequences consisting of members of $\omega^{<\omega}$. 
Lemma 3.1 [BL]. A space $X$ is weakly submetacompact if and only if every open cover $\mathscr{U}$ of $X$ has an open refinement $\cup_{n<\omega} \mathscr{V}_{n}$ such that one can choose some $n<\omega$ with $\operatorname{ord}\left(x, \mathscr{V}_{n}\right)=1$.

Lemma 3.1 seems to be more convenient to use rather than Definition 2.1.

Proof of Theorem 2.3. Let $s$ be a winning strategy for Player I in $G(D C, X)$. Let $\mathscr{O}$ be any open cover of $X$. We may assume without loss of generality that $\mathscr{O}$ is closed under finite unions.

Let $A(\varnothing)=\{\varnothing\}, G(\varnothing)=\varnothing$ and $H(\varnothing)=R(\varnothing)=X \times Y$. Let $\mathscr{G}(\varnothing)=\{\varnothing\}$ and $\mathscr{H}(\varnothing)=\mathscr{R}(\varnothing)=\{X \times Y\}$.

For each $t=\left(\tau_{1}, \ldots, \tau_{n}\right) \in\left(\omega^{<\omega}\right)^{<\omega}$, we shall construct an index set $A(t)$ consisting of $n$-tuple sequences and the three collections $\mathscr{G}(t), \mathscr{H}(t)$ and $\mathscr{R}(t)$ of rectangles in $X \times Y$, satisfying the following conditions (1)-(9):

(1) $\mathscr{G}(t)=\{G(a): a \in A(t)\}$ and $\mathscr{H}(t)=\{H(a): a \in A(t)\}$ are collections of open rectangles, and $\mathscr{R}(t)=\{R(a): a \in A(t)\}$ is a collection of closed $\times$ open rectangles.

(2) If $z \in H(a) \cap R(a)$ for some $a \in A(t)$ and $\operatorname{ord}(z, \mathscr{H}(t))=1$, then there are some $\tau \in \omega^{<\omega}$ and some $\alpha$ such that $a \oplus \alpha \in A(t \oplus \tau)$, either $z \in H(a \oplus \alpha) \cap R(a \oplus \alpha)$ or $z \in G(a \oplus \alpha)$, and $\operatorname{ord}(z, \mathscr{H}(t \oplus \tau))=1$.

For each $a \in A(t)$,

(3) $a_{-} \in A\left(t_{-}\right)$,

(4) $G(a) \subset 0$ for some $0 \in \mathscr{O}$,

(5) $G(a) \subset H(a)$,

(6) $H(a)^{\prime \prime}=R(a)^{\prime \prime}$ if $H(a)$ and $R(a)$ are nonempty,

(7) $H(a) \subset H\left(a_{-}\right)$,

(8) $R(a) \subset R\left(a_{-}\right)$,

(9) $s\left(R\left(a_{-}\right)^{\prime}\right) \cap R(a)^{\prime}=\varnothing$.

Assume that we have already constructed $A(t), \mathscr{G}(t), \mathscr{H}(t)$ and $\mathscr{R}(t)$ satisfying (1)-(9) for each $t \in \bigcup_{i \leq n}\left(\omega^{<\omega}\right)^{i}$.

Pick any $t=\left(\tau_{1}, \ldots, \tau_{n}\right) \in\left(w^{<\omega}\right)^{n}$ and any $a=\left(\alpha_{1}, \ldots, \alpha_{n}\right) \in A(t)$. There is a discrete collection $\left\{C_{\lambda}: \lambda \in \Lambda(a)\right\}$ of compact sets in $X$ such that $s\left(R(a)^{\prime}\right)=\cup\left\{C_{\lambda}: \lambda \in \Lambda(a)\right\}$. Since $X$ is regular submetacompact, we can take a collection $\left\{W_{\lambda, m}: \lambda \in \Lambda(a)\right.$ and $\left.m<\omega\right\}$ of open sets in $X$ such that

(i) $C_{\lambda} \subset W_{\lambda, m} \subset \bar{W}_{\lambda, m} \subset X \backslash \cup\left\{C_{\mu}: \mu \in \Lambda(a)\right.$ with $\left.\mu \neq \lambda\right\}$ for each $\lambda \in \Lambda(a)$ and $m<\omega$.

(ii) for each $x \in X$, one can choose some $m(x)<\omega$ such that

$$
\operatorname{ord}\left(x,\left\{W_{\lambda, m(x)}: \lambda \in \Lambda(a)\right\}\right)<\omega .
$$

Since $Y$ is weakly submetacompact, it follows from Lemma 3.1 that for each $\lambda \in \Lambda(a)$ and $m<\omega$ there is a collection

$$
\left\{U_{\delta} \times V_{\delta}: \delta \in \Delta_{m, n}(a ; \lambda) \quad \text { and } n<\omega\right\}
$$


of open rectangles in $X \times Y$, satisfying

(iii) $C_{\lambda} \subset U_{\delta} \subset W_{\lambda, m}$,

(iv) $U_{\delta} \times V_{\delta} \subset 0$ for some $0 \in \mathscr{O}$,

(v) $\cup\left\{V_{\delta}: \delta \in \Delta_{m, n}(a ; \lambda)\right.$ and $\left.n<\omega\right\}=R(a)^{\prime \prime}$,

(vi) for each $y \in R(a)^{\prime \prime}$, there is some $n(y)<\omega$ such that

$$
\operatorname{ord}\left(y,\left\{V_{\delta}: \delta \in \Delta_{m, n(y)}(a ; \lambda)\right\}\right)=1 \text {. }
$$

Let $\Lambda(a)$ be a set well-ordered by <. Pick any $\tau=\left(m, n_{1}, \ldots, n_{k}\right) \in$ $\omega^{<\omega} \backslash \omega^{1}$. Put

$$
A(t \oplus \tau)=\left\{a \oplus \alpha: \alpha=\left(\delta_{1}, \ldots, \delta_{k}\right), \delta_{i} \in \Delta_{m, n_{i}}\left(a ; \lambda_{i}\right), \lambda_{i} \in \Lambda(a)\right.
$$

for each $i \leq k$ with $\lambda_{1}<\cdots<\lambda_{k}$ and $\left.a \in A(t)\right\}$.

Pick any $a \oplus \alpha \in A(t \oplus \tau)$, where $\alpha=\left(\delta_{1}, \ldots, \delta_{k}\right), \delta_{i} \in \Delta_{m, n_{i}}\left(a ; \lambda_{i}\right)$, $\lambda_{i} \in \Lambda(a)$ for each $i \leq k$. Let

$$
\begin{gathered}
W(a \oplus \alpha)=W_{\lambda_{1}, m} \cap \cdots \cap W_{\lambda_{k}, m} \cap H(a)^{\prime}, \\
U(a \oplus \alpha)=U_{\delta_{1}} \cup \cdots \cup U_{\delta_{k}} \quad \text { and } \quad V(a \oplus \alpha)=V_{\delta_{1}} \cap \cdots \cap V_{\delta_{k}} .
\end{gathered}
$$

Moreover, let

$$
\begin{aligned}
G(a \oplus \alpha) & =(U(a \oplus \alpha) \cap W(a \oplus \alpha)) \times V(a \oplus \alpha), \\
H(a \oplus \alpha) & =W(a \oplus \alpha) \times V(a \oplus \alpha), \\
R(a \oplus \alpha) & =\left[\overline{\left.(W(a \oplus \alpha) \backslash U(a \oplus \alpha)) \cap R(a)^{\prime}\right] \times V(a \oplus \alpha) .}\right.
\end{aligned}
$$

Pick any $\tau=(m) \in \omega^{1}$. Put $A(t \oplus(m))=\left\{a \oplus \theta_{m}: a \in A(t)\right\}$. For each $a \oplus \theta_{m} \in A(t \oplus(m))$, let $G\left(a \oplus \theta_{m}\right)=\varnothing, H\left(a \oplus \theta_{m}\right)=H(a)$ and

$$
R\left(a \oplus \theta_{m}\right)=\left(R(a)^{\prime} \backslash \cup\left\{W_{\lambda, m}: \lambda \in \Lambda(a)\right\}\right) \times R(a)^{\prime \prime} .
$$

Now we have constructed $\mathscr{G}(t \oplus \tau)=\{G(b): b \in A(t \oplus \tau)\}, \mathscr{H}(t \oplus \tau)=$ $\{H(b): b \in A(t \oplus \tau)\}$ and $\mathscr{R}(t \oplus \tau)=\{R(b): b \in A(t \oplus \tau)\}$ for each $t \oplus \tau \in$ $\left(\omega^{<\omega}\right)^{n+1}$. It is easy to check that all conditions except (2) are satisfied.

Let $z=(x, y) \in X \times Y$. Take $t \in\left(\omega^{<\omega}\right)^{<\omega}$ and $a \in A(t)$. Assume that $z \in H(a) \cap R(a)$ and $\operatorname{ord}(z, \mathscr{H}(t))=1$. Note that $z \notin H(c)$ for each $c \in A(t)$ with $c \neq a$.

The case of $x \notin \cup\left\{W_{\lambda, m}: \lambda \in \Lambda(a)\right\}$ for some $m<\omega$ : Then it is obvious that $a \oplus \theta_{m} \in A(t \oplus(m)), z \in H\left(a \oplus \theta_{m}\right) \cap R\left(a \oplus \theta_{m}\right)$ and $\operatorname{ord}(x, \mathscr{H}(t \oplus(m)))=1$.

The case of $x \in \bigcap_{m<\omega}\left(\cup\left\{W_{\lambda, m}: \lambda \in \Lambda(a)\right\}\right)$ : By (ii), we can choose $m<\omega$ such that $1 \leq \operatorname{ord}\left(x,\left\{W_{\lambda, m}: \lambda \in \Lambda(a)\right\}\right)<\omega$. Let

$$
\left\{\lambda \in \Lambda(a): x \in W_{\lambda, m}\right\}=\left\{\lambda_{1}, \ldots, \lambda_{k}\right\} \text {, where } \lambda_{1}<\cdots<\lambda_{k} .
$$

By $y \in R(a)^{\prime \prime}$ and (vi), for each $i \leq k$ we can choose $n_{i}<\omega$ such that

$$
\operatorname{ord}\left(y,\left\{V_{\delta}: \delta \in \Delta_{m, n_{i}}\left(a ; \lambda_{i}\right)\right\}\right)=1 \text {. }
$$

Then there is a $\delta_{i} \in \Delta_{m, n_{i}}\left(a ; \lambda_{i}\right)$ such that

$$
y \in V_{\delta_{i}} \backslash \cup\left\{V_{\delta}: \delta \in \Delta_{m, n_{i}}\left(a ; \lambda_{i}\right) \quad \text { with } \quad \delta \neq \delta_{i}\right\}
$$


for which $i \leq k$. Now, let $\tau=\left(m, n_{1}, \ldots, n_{k}\right) \in \omega^{<\omega} \backslash \omega^{1}$ and $\alpha=\left(\delta_{1}, \ldots, \delta_{k}\right)$. Then $a \oplus \alpha \in A(t \oplus \tau)$. Observe that

$$
\begin{aligned}
z & =(x, y) \in\left(\bigcap_{i \leq k} W_{\lambda_{i}, m} \cap H(a)^{\prime}\right) \times\left(\bigcap_{i \leq k} V_{\delta_{i}}\right) \\
& =W(a \oplus \alpha) \times V(a \oplus \alpha)=H(a \oplus \alpha) \in \mathscr{H}(t \oplus \tau) .
\end{aligned}
$$

If $x \in U_{\delta_{1}} \cup \cdots \cup U_{\delta_{k}}=U(a \oplus \alpha)$, then we have

$$
z=(x, y) \in(W(a \oplus \alpha) \cap U(a \oplus \alpha)) \times V(a \oplus \alpha)=G(a \oplus \alpha) .
$$

If $x \notin U(a \oplus \alpha)$, then we have

$$
z=(x, y) \in\left[(W(a \oplus \alpha) \backslash U(a \oplus \alpha)) \cap R(a)^{\prime}\right] \times V(a \oplus \alpha) \subset R(a \oplus \alpha) .
$$

Pick any $b \in A(t \oplus \tau)$ with $b \neq a \oplus \alpha$. If $b_{-} \neq a$, then $z \notin H(b)$. For $b_{-} \in A(t)$, so $z \notin H\left(b_{-}\right) \supset H(b)$. Assume that $b_{-}=a$. Let $b=a \oplus \beta$, where $\beta=\left(\epsilon_{1}, \ldots, \epsilon_{k}\right), \epsilon_{i} \in \Delta_{m, n_{i}}\left(a ; \mu_{i}\right)$ and $\mu_{i} \in \Lambda(a)$ for each $i \leq k$ with $\mu_{1}<\cdots<\mu_{k}$. If $\left(\lambda_{1}, \ldots, \lambda_{k}\right) \neq\left(\mu_{1}, \ldots, \mu_{k}\right)$, then we can find $i \leq k$ such that $\mu_{i} \neq \lambda_{j}$ for each $j \leq k$. Then $x$ is not in $W_{\mu_{i}, m}$. Assume that $\left(\lambda_{1}, \ldots, \lambda_{k}\right)=\left(\mu_{1}, \ldots, \mu_{k}\right)$. Since $\alpha$ and $\beta$ must be distinct, we can find $i \leq k$ such that $\delta_{i}, \epsilon_{i} \in \Delta_{m, n_{i}}\left(a ; \lambda_{i}\right)$ and $\delta_{i} \neq \epsilon_{i}$. Then $y$ is not in $V_{\epsilon_{i}}$. So, in either case, we have

$$
z=(x, y) \notin W_{\mu_{i}, m} \times V_{\epsilon_{i}} \supset W(a \oplus \beta) \times V(a \oplus \beta)=H(a \oplus \beta)=H(b) .
$$

Hence $\operatorname{ord}(z, \mathscr{H}(t \oplus \tau))=1$. Thus (2) is satisfied. After all, the conditions (1)-(9) are satisfied.

Now, we take the countable family $\left\{\mathscr{G}(t): t \in\left(\omega^{<\omega}\right)^{<\omega}\right\}$ of collections of open rectangles in $X \times Y$. By (1) and (4), each $G \in \mathscr{G}(t)$ is contained in some $0 \in \mathscr{O}$. It suffices to show that for each $z \in X \times Y$ there is some $t \in\left(\omega^{<\omega}\right)^{<\omega}$ such that $\operatorname{ord}(z, \mathscr{G}(t))=1$. Assuming the contrary, pick some $z_{0} \in X \times Y$ such that $\operatorname{ord}\left(z_{0}, \mathscr{G}(t)\right) \neq 1$ for each $t \in\left(\omega^{<\omega}\right)^{<\omega}$.

Claim. There are two sequences $\left\{t_{n}\right\}_{n \geq 0}$ and $\left\{a_{n}\right\}_{n \geq 0}$ such that for each $n<$ $\omega$

(vii) $t_{n} \in\left(\omega^{<\omega}\right)^{<\omega}$ with $\left(t_{n}\right)_{-}=t_{n-1}$

(viii) $a_{n} \in A\left(t_{n}\right)$ with $\left(a_{n}\right)_{-}=a_{n-1}$,

where $t_{0}=\varnothing$, where $a_{0}=\varnothing$,

(ix) $z_{0} \in H\left(a_{n}\right) \cap R\left(a_{n}\right)$

(x) $\operatorname{ord}\left(z_{0}, \mathscr{H}\left(t_{n}\right)\right)=1$

The case of $n=0$ is clear. Assume that we have already chosen $t_{0}, \ldots, t_{n}$ and $a_{0}, \ldots, a_{n}$ as above. By (2), we can choose some $t_{n+1} \in\left(\omega^{<\omega}\right)^{<\omega}$ and some $a_{n+1} \in A\left(t_{n+1}\right)$, satisfying (vii), (viii) and (x) for $n+1$. Assume that (ix) for $n+1$ is not satisfied. Then (2) assures that $z_{0} \in G\left(a_{n+1}\right) \in \mathscr{G}\left(t_{n+1}\right)$. By (5) we have

$$
1 \leq \operatorname{ord}\left(z_{0}, \mathscr{G}\left(t_{n+1}\right)\right) \leq \operatorname{ord}\left(z_{0}, \mathscr{H}\left(t_{n+1}\right)\right)=1 .
$$

Hence, $\operatorname{ord}\left(z_{0}, \mathscr{G}\left(t_{n+1}\right)\right)=1$. This is a contradiction. 
It follows from (viii), (8) and (9) that $R\left(a_{n+1}\right)^{\prime} \subset R\left(a_{n}\right)^{\prime}$ and $s\left(R\left(a_{n}\right)^{\prime}\right) \cap$ $R\left(a_{n+1}\right)^{\prime}=\varnothing$ for each $n<\omega$. By the choice of $s$, we have $\bigcap_{n<\omega} R\left(a_{n}\right)^{\prime}=\varnothing$. Hence $\bigcap_{n<\omega} R\left(a_{n}\right)=\varnothing$. This contradicts $z_{0} \in \bigcap_{n<\omega} R\left(a_{n}\right)$ in (ix). The proof of Theorem 2.3 is completed.

Added in proof. Recently, Gruenhage and the author have given an affirmative answer to our Question 1.1 in the paper titled "A filter property of submetacompactness and its application to products," which will appear in Topology and its Applications.

\section{REFERENCES}

[B] D. K. Burke, Covering properties, Handbook of set-theoretic topology (Kunen and J. E. Vaughan, editors), North-Holland, Amsterdam, 1984, pp. 347-422.

[BL] H. B. Bennett and D. J. Lutzer, A note on weak $\theta$-refinability, Gen. Topology Appl. 2 (1972), 49-54.

[GT] F. Galvin and R. Telgársky, Stationary strategies in topological games, Topology Appl. 22 (1986), 51-69.

[K] Y. Katuta, On spaces which admit closure-preserving covers by compact sets, Proc. Japan Acad. 50 (1974), 826-828.

[L] D. J. Lutzer, Another property of the Sorgenfrey line, Compositio Math. 24 (1972), 359-363.

[N] K. Nagami, L-spaces, Fund. Math. 65 (1969), 169-192.

[P] T. C. Przymusinski, Normality and paracompactness in finite and countable Cartesian products, Fund. Math. 105 (1980), 87-104.

[PJ] H. B. Potoczny and H. Junnila, Closure-preserving families and metacompactness, Proc. Amer. Math. Soc. 53 (1975), 523-529.

[T] R. Telgársky, Spaces defined by topological games, Fund. Math. 88 (1975), 193-223.

[WW] J. M. Worrell and H. H. Wicke, Characterizations of developable topological spaces, Canad. J. Math. 17 (1965), 820-830.

[ $\left.Y_{1}\right]$ Y. Yajima, Topological games and products II, Fund. Math. 117 (1983), 47-60.

[ $\mathrm{Y}_{2}$ ] _ , Topological games and products III, Fund. Math. 117 (1983), 223-238.

Department of Mathematics, Kanagawa University, Yokohama 221, Japan

Division of Mathematics, Foundations, ANalysis, and Topology, Auburn University, Alabama 36849 